\title{
Serum squamous cell carcinoma antigen in psoriasis: a potential quantitative biomarker for disease severity
}

\author{
Ziwen Sun ${ }^{1,2,5}$, Xiaomin Shi ${ }^{3,5}$, Yun Wang ${ }^{2}$, Suzhen $\mathrm{Ji}^{2}$, Yi Zhao ${ }^{4 *}$ \\ 1 - Shanghai dermatology Hospital ( No.1278, BaoDe road, Shanghai), Shanghai 200443 China; 2. Department of Dermatology \\ and Venereology, Peking University First hospital, Being 100191, China; 3. Department of Clinical Laboratory, Peking University, \\ First hospital, Being 100034, China; 4.Department of Dermatology, Beijing Tsinghua Changgung Hospital, Tsinghua University, \\ Beijing 102218, China; 5. These authors contributed equally to this work. *Corresponding author: Zhao Yi, zhaoyi@bjmu.edu.cn
}

\section{Objectives:}

To explore serum biomarkers quantitatively in association with disease severity and treatment response in psoriasis patients.

\section{Methods:}

15 psoriasis patients were treated with adalimumab. At different visits before and after treatment, quantitative body surface area (qBSA) was obtained from standardized digital body images of the patients, and psoriasis area severity index (PASI) was also monitored. Serum squamous cell carcinoms antigen (SCCA) and tumor necrosis factor-alpha (TNF- $\alpha$ ) were detected by using Microparticle Enzyme Immuno Assay and Enzyme Linked Immunosorbent Assay. The serum biomarkers were also tested in healthy volunteers as normal control. Receiver operating characteristic (ROC) curve analysis was used to explore the optimal cut-off point of SCCA to differentiate mild and moderate-to-severe psoriasis.

\section{Results:}

1. Efficacy. Figure 1. PASI (a, b) and qBSA (c, d) of psoriasis patients before and after adalimumab treatment. ADA, Patients received adalimumab; PBO, Patients received placebo.
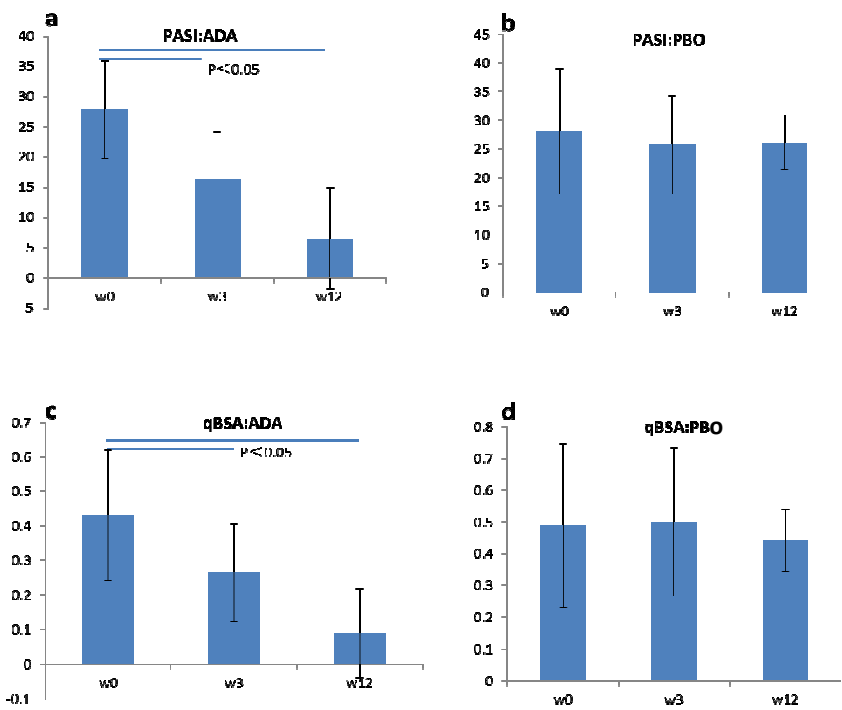

2. The serum SCCA level in psoriasis group was significantly higher $(P<0.05)$ than in the normal control group. Table1. The level of TNF- $\alpha$ and SCCA in controls and psoriasis patients.

\begin{tabular}{|c|c|c|c|c|}
\hline & \multicolumn{2}{|c|}{ TNF- $\alpha(\mathrm{ng} / \mathrm{ml})$} & \multicolumn{2}{|c|}{$\mathrm{SCCA}(\mathrm{ng} / \mathrm{ml})$} \\
\hline & Mean \pm sd & Range & Mean $\pm s d$ & Range \\
\hline Controls & $2.16 \pm 0.84$ & $0.9-3.3$ & $1.34 \pm 0.52$ & $0.6-2.2$ \\
\hline Psoriasis" & $3.33 \pm 4.57$ & $0.9-18.1$ & $17.45 \pm 19.90^{*}$ & $3.4-69.4$ \\
\hline
\end{tabular}

\# before treatment; ${ }^{*} \mathrm{P}<0.05$ in comparison with controls.
3. After treatment, the serum SCCA levels were significantly decreased $(P<0.05)$. Table 2 . Serum SCCA levels at three visits

\begin{tabular}{|c|c|c|c|c|}
\hline Group & $\mathrm{N}$ & Week 0 & Week 3 & Week 12 \\
\hline ADA & 12 & $18.5 \pm 21.2$ & $11.4 \pm 17.9$ & $3.7 \pm 5.4^{*}$ \\
\hline PBO & 3 & $7.6 \pm 6.6$ & $6.1 \pm 1.4$ & $6.2 \pm 0.4$ \\
\hline
\end{tabular}

${ }^{*} \mathrm{P}<0.05$ in comparison with week 0. ADA adalimumab, $\mathrm{PBO}$ placebo

4. SCCA level was well correlated to PASI and qBSA. Figure 2. Scatter plot of SCCA-PASI (a) and SCCA-qBSA (b)
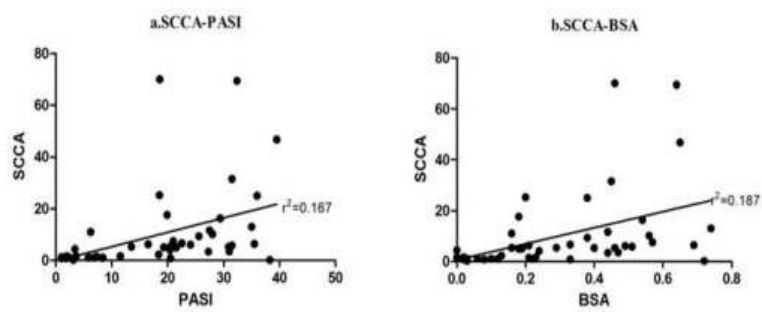

5. In ROC analysis, when taking $P A S I=10$ or $q B S A=10$ as the threshold, an optimal cut-off point of SCCA was found at $2.0 \mathrm{ng} / \mathrm{mL}$ with the highest Youden's Index. Fig. 3 ROC curves of serum SCCA-PASI (a) or SCCA-qBSA (b) and serum SCCA level when setting PASI=10 (c) or $q B S A=10$ (d) as the threshold.
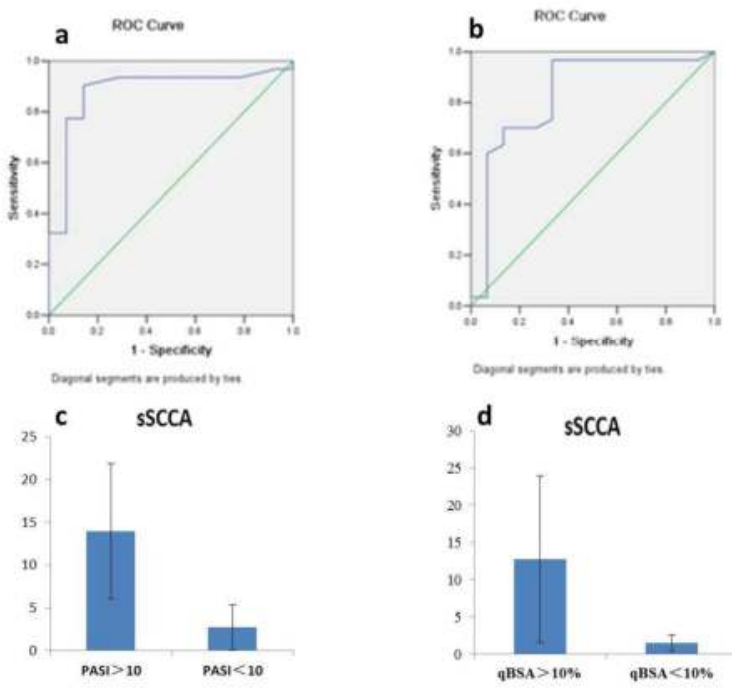

Conclusion:

Serum SCCA might be a useful quantitative biomarker for psoriasis disease severity. 